\title{
Assistência fisioterapêutica em prematuros com Síndrome do Desconforto Respiratório: uma revisão de literatura
}

A Síndrome do Desconforto Respiratório (SDR) é um distúrbio que acomete principalmente os recém-nascidos prematuros pela imaturidade pulmonar, resultante da deficiência ou inativação do surfactante. 0 surfactante é uma substância lipoproteica produzida pelas células pneumócitos tipo Il e armazenado nos corpos lamelares para, posteriormente, ser liberado no espaço alveolar, sintetizado a partir da $200^{\circ}$ semana de gestação, com pico de produçãa A çăo do surfactante é diminuir a tensão superficial dos alvéolos, evitando o colabamento na expiraçăo. Quanto menor a idade gestacional de nascimento, maior o risco de desenvolver a SDR. A prematuridade e a imaturidade do sistema respiratório levam a maior necessidade de suporte ventilatório invasivo, oxigenioterapia, assistencicia fisioterapéutica e internação na unidade de terapia intensiva neonatal. A fisioterapia e vigilância nos ajustes dos parâmetros da ventilação mecânica invasiva e não invasiva. Este estudo tem o objetivo de realizar uma revisão bibliográfica para identificar as estratégias terapêuticas respiratórias utilizadas pelo fisioterapeuta na assistência ao recém-nascido prematuro com Síndrome do Desconforto Respiratório. Para este fim, fizemos um levantamento de artigos científicos em bases de dados eletrônicos como ScieLO, LLLACS, Google Acadêmico e PubMed, através dos cognatos 'Síndrome do Desconforto Respiratório', 'prematuros', 'unidade de terapia intensiva neonatal', 'fisioterapia' e 'ventilação mecânica', e seus correlatos em inglês 'Respiratory Distress Syndrome', 'premature infants', 'neonatal intensive care unit', e 'physical therapy and mechanical ventilation'. A assistência fisioterapêutica a neonatos prematuros com SDR na UTIN é imprescindivel para o sucesso da terapêutica e melhora progressiva da posicionamento e cuidados com a ocorrência de sequelas como hemorragia intraperiventricular e displasia broncopulmonar que podem comprometer o desenvolvimento neuropsicomotor e qualidade de vida após alta hospitalar.

Palauras-chave: Síndrome do Desconforto Respiratório; Prematuros; Unidade de Terapia Intensiva Neonatal; Fisioterapia; Ventilação Mecânica.

\section{Physiotherapeutic care in premature infants with Respiratory Distress Syndrome: a literature review} \begin{abstract}
Respiratory Distress Syndrome (RDS) is a disorder that mainly affects premature newborns due to pulmonary immaturity resulting from the deficiency or inactivation of the surfactant. The surfactant is a lipoprotein substance
produced by the type II pneumocyte cells and stored in the lamellar bodies and later released into the alveolar space, synthesized from the 20 th week of gestation, with peak production around 35 weeks. The function of the surfactant is to reduce the superficial tension of the alveoli, avoiding the collapse in the expiration. The lower the gestational age at birth, the greater the risk of developing RDS. The prematurity and immaturity of the respiratory system lead to a greater need for invasive ventilatory support, oxygen therapy, physiotherapeutic assistance and hospitalization in the neonatal intensive care unit. Respiratory physiotherapy plays an important role in the maintenance of airway permeability, prevention of respiratory complications, promotion of bronchial hygiene, optimization of pulmonary reexpansion, adequate positioning and monitoring of the parameters of invasive and non-invasive mechanical ventilation. This study aims to perform a bibliographic review to identify the respiratory therapy strategies used by the physiotherapist in the care of the premature newborn with Respiratory Distress Syndrome. To this end, we did a survey of scientific articles in electronic databases such as SciELO, LLLACS, Google Academic and PubMed, through the cognates 'Respiratory Distress 'Syndrome', 'premature neonates', 'neonata

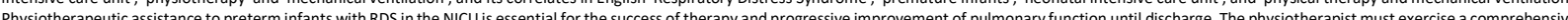
Physiotherapeutic assistance to preterm infants with RDS in the Nic is essential forthe success of therapy and progressive improvement of pulmonary function until discharge. The physiotherapist must exercise a comprehensive (ischarge.
\end{abstract}

Keywords: Respiratory Distress Syndrome; Premature; Neonatal Intensive Care Unit; Physiotherapy; Mechanical ventilation.

Topic: Fisioterapia

Reviewed anonymously in the process of blind peer

Simone Amancio Teles

Faculdade Guaraí, Brasil

http://lattes.cnpq.br/0591590391105455

http://orcid.org/0000-0001-5934-0219

simon-fisio@hotmail.com

Marineth Ferreira de Carvalho Teixeira (iD)

Faculdade Guaraí, Brasil

http://lattes.cnpq.br/9930544738694476

http://orcid.org/0000-0001-8074-9166

marinethcarvalho@ymail.com

Daniela Maristane Vieira Lopes Maciel (iD)

Faculdade Guaraí, Brasil

http://lattes.cnpq.br/0591590391105455

http://orcid.org/0000-0001-5934-0219

daniela.marciel@iescfag.edu.br

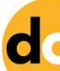

DOI: 10.6008/CBPC2236-9600.2018.002.0005
Received: 08/08/2019

Approved: 19/08/2019
Referencing this:

SOBRENOME, N. N.; SOBRENOME, N. N.. Assistência fisioterapêutica em prematuros com Síndrome do Desconforto Respiratório: uma revisão de literatura. Scire Salutis, v.8, n.2, p.43-53, 2018. DOI: http://doi.org/10.6008/CBPC2236-9600.2018.002.0005 


\section{INTRODUÇÃO}

Nas últimas décadas, observaram-se grandes avanços, não só no conhecimento dos processos que envolvem a maturação pulmonar fetal, mas também nos cuidados de suporte aos recém-nascidos pré-termo (RNPT) de peso baixo (ROSO et al., 2014; ALDANA-AGUIRRE et al., 2017). Desde o 'esquema de Usher' dos anos 60 , até o uso de suporte ventilatório mais sofisticados como a ventilação de alta frequência e dos surfactantes exógenos, na década de 90, gerando uma grande contribuição no aumento da sobrevida dos neonatos com a síndrome do desconforto respiratório (SDR) (ALDANA-AGUIRRE et al., 2017).

Com isso, o aumento da sobrevida dos RNPT com SDR pode ser atribuído à associação da terapia de corticoide no período gestacional, surfactante exógeno pós-natal e principalmente aos avanços no suporte ventilatório nas unidades de terapia intensivas neonatais (UTIN) (UTYAMA et al., 2016). Os RNPT e de baixo peso são mais vulneráveis a sofrerem com fatores de risco pós-natais tais quais hemorragia intraperiventricular, síndrome do desconforto respiratório, infecções, displasia broncopulmonar (DBP), distúrbios metabólicos, hematológicos e gastrintestinais (PICCOLI et al., 2012). Devido à imaturidade do sistema respiratório, os RNPT apresentam altos riscos de desenvolverem complicações respiratórias com necessidade de suporte ventilatório invasivo, e com isso a necessidade de intervenção fisioterapêutica respiratória (ALMEIDA et al., 2013).

A SDR é um distúrbio que acomete principalmente os RNPT resulta da imaturidade pulmonar, resultante da deficiência e/ou inativação do surfactante (ALDANA-AGUIRRE et al., 2017), desencadeando alterações alveolares importantes como microatelectasias progressivas gerando diminuição da complacência pulmonar, liberação de uma cascata inflamatória desencadeando lesão do epitélio alveolar e endotélio capilar, e consequentemente formação da membrana hialina e extravasamento de líquido rico em proteínas no espaço alveolar (RUSHEL et al., 2014).

O surfactante é uma substância lipoprotéica produzida pelas células pneumócitos tipo II e armazenado nos corpos lamelares para, posteriormente, ser liberado no espaço alveolar (SILVA et al., 2009), sintetizado a partir da 20오 semana de gestação, com pico de produção por volta da 35오 semana. A função do surfactante é diminuir a tensão superficial dos alvéolos, evitando o colabamento na expiração (RUSHEL et al., 2014).

A SDR é mais comum no sexo masculino e em recém-nascidos com idade gestacional menor que 37 semanas, quanto menor o tempo de gestação maior a chance do recém-nascido (RN) desenvolver esta síndrome (SOUZA et al., 2013). Cerca de 50\% dos óbitos neonatais são desencadeados por distúrbios respiratórios, 80 a $90 \%$ desses casos acontecem na primeira semana de vida e 30\% associa-se com idade gestacional inferior a 30 semanas (RUSCHEL et al., 2014; SOUZA et al., 2013).

Com isso, a fisioterapia respiratória tem papel importante na manutenção da permeabilidade das vias aéreas, procurando prevenir as complicações respiratórias, otimizando o mecanismo de depuração mucociliar prevenindo o acúmulo de secreção (ROSO et al., 2014), aperfeiçoando o suporte ventilatório e contribuindo para diminuição do trabalho respiratório (VASCONCELOS et al., 2011). O objetivo deste estudo 
é realizar uma revisão bibliográfica para identificar as estratégias terapêuticas respiratórias utilizadas pelo fisioterapeuta na assistência ao recém-nascido prematuro com síndrome do desconforto respiratório.

\section{METODOLOGIA}

O estudo caracteriza-se como revisão de literatura, baseado em análise de artigos científicos publicados entre 2007 e 2017. Para a elaboração do estudo, foram utilizadas as bases de dados como Literatura Latina Americana em Ciência de Saúde (LILACS), Scientific Electronic Library Online (SciELO), Google Acadêmico e PubMed. Os Descritores em Ciências da Saúde (DCS) utilizados foram: síndrome do desconforto respiratório, prematuros, unidade de terapia intensiva neonatal, fisioterapia e ventilação mecânica e seus correlatos na língua inglesa respiratory distress syndrome, premature infants, neonatal intensive care unit, physical therapy and mechanical ventilation. Os critérios de inclusão determinado foram artigos em língua portuguesa e inglesa relacionados ao tema e que estivessem nos anos de publicação determinados, sendo selecionado 69 artigos e destes 27 excluídos.

\section{DISCUSSÃO TEÓRICA}

A terapêutica assistencial ao RN com SDR deve ser em regime de cuidados intensivos e estabelecida o mais rápido possível após o nascimento, sendo iniciada ainda na sala de parto, a fim de possibilitar a redução da gravidade e complicações da doença. A assistência fisioterapêutica visa tanto à expansão das áreas não ventiladas quanto evitar que novas unidades alveolares colabem comprometendo as áreas de trocas gasosas, oferendo assim suporte ventilatório adequado, manobras de higiene brônquica e reexpansão pulmonar (BITENCOURT, 2017).

Além do suporte ventilatório necessário pela imaturidade pulmonar o fisioterapeuta deve preocuparse com a vulnerabilidade dos RNPT e de baixo peso, principalmente os menores de 1500g, de desenvolverem hemorragia intraperiventricular, pela fragilidade da matriz germinativa e leito vascular, durante o curso das primeiras 72 horas. Devendo ajustar sua conduta para garantir ao RNPT com SDR manuseio mínimo, aspirações se necessário, controle da luminosidade e ruídos dentro da UTIN, estimular também, o apoio familiar para fortalecer o vínculo entre a família e o RN (CABRAL et al., 2014).

O manuseio deve ser rápido, preciso, gentil, cuidadoso e delicado, evitando movimentos bruscos. Tais procedimentos minimizam o estresse e a hipotermia, uma vez que uma das maiores dificuldades do RNPTE é manter sua temperatura corpórea. Os horários de manipulações devem ser agrupados, ou seja, de 4/4 horas, portanto no momento da manipulação devem-se realizar todos os procedimentos necessários, em seguida deixá-lo em repouso e tranquilo (DELLAQUA et al., 2012).

Algumas estratégias de fisioterapia respiratória vêm sendo utilizadas com sucesso como: oxigenoterapia, bag Squeezing, drenagem postural, reexpansão torácica, AFE, posição prona, aspiração de vias aérea, reequilíbrio toracoabdominal (ROUSSENQS et al., 2013), ventilação de alta frequência (VAFO) (FIORETTO et al., 2009; NONA et al., 2009; QUEIROZ et al., 2015). 


\section{Oxigenoterapia}

A oxigenioterapia complementar tem papel importante para o RNPT, porém, deve ser utilizado de forma cautelar, a fim de evitar os efeitos deletérios induzidos pelo oxigênio $\left(\mathrm{O}_{2}\right)$ (BERNADES et al., 2013). A exposição a elevados níveis de concentrações de oxigênio pode levar a alterações na retina, no trato respiratório, lesão do epitélio pulmonar, no espaço pleural e nos septos alveolares (JÚNIOR et al., 2014).

A estratégia ventilatória deve ser ofertada de forma gentil e eficaz, observando sempre suas indicações e contraindicações antes de administrá-la. $\mathrm{O}$ ajuste das frações de oxigênio $\left(\mathrm{FiO}_{2}\right)$ deve ser criteriosas e favorece as trocas gasosas mantendo a saturação periférica de oxigênio num valor desejável (CAVAGNOLI et al., 2014). A saturação de oxigênio preconizada pela literatura diz que deve girar em torno de $90-94 \%$. Sendo assim, se o neonato está recebendo suporte de oxigênio suplementar e apresenta saturação maior que 95\%, essa oferta de oxigênio deve ser prontamente reduzida (CAVAGNOLI et al., 2014). É de suma importância para os fisioterapeutas à avaliação desses neonatos, como o monitoramento dos níveis de oxigênio suplementar que está sendo ofertado, monitorar a $\mathrm{FiO}_{2}$, através da oximetria de pulso ou gasometria arterial (BERNADES et al., 2013; CAVAGNOL et al., 2014).

\section{Associação da CPAP com Surfactante Exógeno}

O uso da ventilação não invasiva (VNI) em recém-nascidos é um recurso tecnológico importante que vem contribuindo para diminuição da mortalidade neonatal e de complicações do suporte ventilatório invasivo prolongado como infecções neonatais, hemorragias, lesão de mucosas, estenoses de traqueia e aumento da incidência de DBP (LEÃO et al., 2013), alteração do fluxo mucociliar normal, infecções (sinusites e pneumonia), aspiração, síndrome do extravasamento de ar (pneumotórax, pneumomediastino e enfisema intersticial), aumento do risco de displasia broncopulmonar, retinopatia da prematuridade, hemorragia intraperiventricular, leucomalácia periventricular e sepse (OLIVEIRA et al., 2009).

A VNI é uma estratégia de suporte ventilatório que através de pressão positiva nas vias aéreas garante aporte de oxigênio e distensão alveolar. Um dos recursos dessa modalidade é a CPAP (Continuous Positive Airways Pressure) que por meio de interfaces faciais dispõem uma pressão contínua nas vias aéreas centímetros de água $\left(\mathrm{cmH}_{2} \mathrm{O}\right)$, esta técnica é segura e muito utilizada em neonatologia através de pronga nasal (YAGUI et al., 2011).

Dentre os efeitos fisiológicos da CPAP estão conservação do surfactante endógeno, melhora da complacência pulmonar, prevenção de colabamento alveolar, aumento da pressão transpulmonar, estabilização das vias aéreas, melhora da capacidade residual funcional (CRF) e melhora da regularidade do ritmo respiratório (SILVA et al., 2010).

A CPAP tem sido apresentando resultados satisfatórios na resolução de complicações respiratórias de RNPT e de baixo peso nas UTIN. Entretanto, a efeitos deletérios como risco de desenvolver síndromes de escape de ar, barotrauma, lesões no septo nasal e distensão abdominal. E para prevenção desses episódios é necessária monitorização criteriosa dos recém-nascidos submetidos a este recurso (SILVA et al., 2010). 
O surfactante pode ser administrado com eficácia em recém-nascidos associado ao CPAP nasal com intubação apenas para administração por via traqueal, seguido de rápida extubação e colocação no CPAP nasal, processo conhecido como INSURE (IN: intubation; SUR: surfactante; e E: extubation). Essa terapêutica objetiva diminuir a exposição do $\mathrm{RN}$ ao suporte ventilatório invasivo com indicação de surfactante exógeno para resolução de SDR na prematuridade (STEVENS et al., 2007).

A administração do surfactante tem sido de grande eficácia para os RNs, reduzindo de forma harmoniosa a mortalidade dos RNPT em 30\% a 40\% (REBELLO et al., 2010). É indicado para RNPT com SDR, além de diminui a ocorrência de pneumotórax, enfisema pulmonar, DBP e óbito neonatal (RUSHEL et al., 2014).

O papel do surfactante é estabilizar os alvéolos, impedindo o colabamento ao final da expiração, diminuindo o trabalho respiratório, aumentando complacência pulmonar (REBELLO et al., 2010), diminuindo o edema pulmonar, melhorando imediatamente a $\mathrm{PaCO}_{2}$ e o débito cardíaco (GEORGE, 2012). É preconizado o manuseio mínimo por pelo menos 12 horas após a administração do surfactante, cabeça na linha média, manter a cabeceira elevada a $30^{\circ} \mathrm{C}$, sem mudança de decúbito e manuseio necessário da equipe multidisciplinar (CABRAL et al., 2014).

Eventos de dessaturação periférica de oxigênio e hipoxemia em RNPT estão associados à mudança de decúbito e ao procedimento de aspiração do tubo endotraqueal após administração de surfactante exógeno. Portanto, as aspirações devem cautelosamente executadas a medida da necessidade, respeitando às 12 horas mínimas de repouso do RN, para evitar também o risco do surfactante se aspirado. Deve-se proceder preventivamente às manobras desobstrutivas e aspirações antes da aplicação do mesmo na via aérea (CABRAL et al., 2014).

O ensaio clínico conduzido por CEYLAN e colaboradores, em 2014, teve a finalidade de comparar se a administração de surfactante precoce e CPAP foi superior à administração tardia de surfactante e ventilação mecânica, em 109 RNPT com SDR, IG de 32 semanas. A intervenção foi realizada em dois grupos, no primeiro o surfactante foi administrado a 61 crianças na sala de parto ou na unidade de terapia intensiva e, posteriormente, colocado CPAP. No segundo grupo o surfactante foi administrado em 48 crianças e posteriormente submetidas a suporte ventilatório invasivo.

O resultado evidenciou que a associação da administração de surfactante precoce e CPAP no tratamento de RNPT com SDR diminuiu acentuadamente o tempo de permanência em suporte ventilatório não invasivo em relação aos que realizaram associaram surfactante exógeno e suporte ventilatório invasivo. Mas não houve impacto significativo na morbimortalidade.

\section{Suporte Ventilatório Invasivo}

Os RNPT admitidos em UTIN rotineiramente necessitam de suporte de oxigênio suplementar, que pode ser não invasiva por meio de uma interface acoplando o RN a VNI ou invasiva por meio de um tubo endotraqueal introduzido acoplando o RN ao ventilador (BERNADES et al., 2013; GONÇALVES et al., 2015). A utilização do suporte ventilatório se faz essencial para aumentar a sobrevida dos RNPT nas UTIN. No entanto, 
essa terapêutica aplicada por períodos prolongados relaciona-se a riscos e complicações, incluindo displasia broncopulmonar e até mesmo a mortalidade neonatal (GONZAGA et al., 2007).

O suporte ventilatório invasivo pode lesionar o epitélio pulmonar, e como também levar ao mau desempenho da função pulmonar, ocorrendo inúmeras disfunções orgânicas múltiplas na tentativa de proporcionar uma troca gasosa adequada (GONÇALVES et al., 2015; FIORETTO et al., 2009). A estratégia ventilatória protetora pulmonar é uma alternativa que promove melhora das trocas gasosas e reduz a lesão pulmonar induzida pela ventilação invasiva com base no uso de volumes correntes baixos e PEEP adequada para uma faixa de melhor complacência pulmonar (ANGHEBEN et al., 2011).

Um recurso ventilatório invasivo muito utilizado no tratamento de RNs com SDR é a ventilação de alta frequência VAFO, que consiste em um modo ventilatório que determina uma Pressão Média das vias aéreas (MAP) suficientemente elevada necessária para expandir os alvéolos colapsados. Essa terapêutica reduz a hiperdistensão pulmonar favorecendo as trocas gasosas (NONA et al., 2009; FIORETTO et al., 2009). Ela utiliza volumes correntes baixos (menores que o espaço morto anatômico) e frequências respiratórias altas (de 600 a 900 inspirações por minuto) (ANGHEBEN et al., 2011).

A VAFO é indicada em três situações explicitas: em caso que visa a reexpansão alveolar, como também em RNs que não obteve sucesso utilizando suporte ventilatório invasivo controlado ou que estão em uso de pressão inspiratória em níveis muito altos, ou em técnica de baixo volume nas síndromes de escape de ar; profilática, que pode sugerir em RNPT, com a finalidade de focalizar a prevenção de lesão no epitélio pulmonar (FIORETTO et al., 2009).

\section{Aspiração de Vias Aéreas}

A aspiração consiste em uma técnica mecânica manual por meio da introdução de uma sonda flexível asséptico na via aérea (VA) e aplicação de pressão superior à da atmosférica no momento de sua retirada para sucção das secreções traqueobrônquicas (VIANA et al., 2016). Em RNPT que estejam submetidos aos cuidados de terapia intensiva, essa técnica é rotineiramente utilizada, tendo em vista a prevenção do acúmulo de secreções endotraqueais, manter vias aéreas pérvias, aumentar o fluxo de oxigênio, melhorar ventilação perfusão (V/Q) (GONÇALVES et al., 2015; VIANA et al., 2016).

Estudos determinam que o tempo de introdução da sonda deva ser o mais breve possível, sendo a mesma retirada com movimentos circulares, a fim de evitar lesões à mucosa traqueal e desconforto do neonato. Sendo de suma importância sua utilização apenas quando necessário, ou seja, quando houver sinais indicativos da presença muco nas VA, sendo que essa avaliação deva ser realizada rigorosamente pelo fisioterapeuta através de ausculta pulmonar bilateral, para evitar que o RN seja submetido a aspirações desnecessárias (MARTINS et al., 2014).

O estudo de intervenção de FIATT e colaboradores, em 2013, relata a reversão total do colapso pulmonar direito (D) em um RNPT com IG de 27 semanas, extremo baixo peso, sendo admitido na UTIN aos 45 minutos de vida devido à prematuridade e a SDR. Quando realizou a aspiração das vias aéreas após execução das manobras respiratórias AFE lenta e compressão-descompressão torácica. Provando assim ser 
um recurso eficaz e necessário após manobras fisioterapêuticas respiratórias a fim de promover a eliminação da secreção pulmonar descolada em RN submetidos a ventilação invasiva.

\section{Bag Squeezing}

O bag Squeezing consiste em um procedimento realizado com o auxílio de uma bolsa de hiperinsuflação pulmonar associada a manobras fisioterapêuticas de vibração e compressão torácica na expiração, comumente usada em UTIN, em pacientes em suporte ventilatório invasivo, visando reverter o colapso alveolar, auxiliar na remoção de secreção e melhorar áreas de trocas gasosas (VIANA et al., 2016).

O estudo prospectivo transversal de Chaves et al. (2013), com 28 RNPT com IG inferior a 35 semanas, estabilidade hemodinâmica, submetidas à VM invasiva e dependente desse recurso aos 28 dias, analisaram os efeitos na frequência cardíaca e respiratória, na saturação periférica de oxigênio, no volume pulmonar inspiratório e expiratório antes, durante e após a manobra de bag Squeezing, utilizando ressuscitador manual com ou sem válvula reguladora da PEEP. O desfecho pode evidenciar que a manobra de bag Squeezing é benéfica para o RNPT, independente do uso de válvula, resultando em melhora significativa da resistência expiratória e aumento do volume pulmonar inspiratório e expiratório.

A revisão de literatura de BURIA, em 2013, analisou e discutiu a segurança da aplicação da técnica de Hiperinsuflação Manual, bag Squeezing. E constatou que os estudos disponíveis não conseguiram demonstrar os benefícios a longo prazo, a segurança da aplicação da manobra e uma padronização da técnica. O ensaio clínico de Steidl et al. (2009), avaliou 9 RNPT, entre 26 e 37 semanas de idade gestacional, em suporte ventilatório invasivo na UTIN, observando melhora progressiva da saturação periférica de oxigênio após manobra de bag Squeezing e invariabilidade da frequência cardíaca e frequência respiratória ao longo do tempo.

\section{Aceleração do Fluxo Expiratório (AFE)}

A AFE é uma técnica não convencional de desobstrução brônquica que pode ser aplicada desde o nascimento, inclusive no RN prematuro, quando existe doença respiratória com obstrução das vias aéreas. A desobstrução é realizada por meio de preensão bimanual, com uma mão envolvendo e comprimindo suavemente a parede anterolateral do tórax do RN durante a expiração, enquanto a outra mão exerce apoio estático no abdome (SÁ et al., 2012).

O rápido aumento da pressão intratorácica devido à compressão abdominotorácica leva à expulsão de secreções. O fisioterapeuta usa as duas mãos. Um colocado no tórax o outro no abdômen serve como contra apoio com os cuidados inerentes aos recém-nascidos. A sincronização das duas mãos garante a permanência da progressão. O movimento torácico abdominal é realizado no momento expiratório pelas mãos do fisioterapeuta (SILVA et al., 2010).

O posicionamento das mãos do fisioterapeuta pode ser variável (uma mão esternal e um suporte dorsal duplo, duas mãos sob as clavículas, uma mão esternal e uma mão abdominal. $\mathrm{O}$ paciente inspira 
profundamente e expira, abra a glote, até o volume residual. Velocidade, força e duração podem variar (SILVA et al., 2010).

Para avaliar os efeitos fisiológicos da técnica AFE lenta, SÁ et al. (2012) propôs um estudo de caráter quantitativo e de intervenção com 8 prematuros, IG entre 28 e 36 semanas, analisando as seguintes variáveis em dois momentos: sinais de angústia respiratória, tipo de respiração, comportamento, coloração da pele, tipo, cor e quantidade de secreção, saturação percutânea de oxigênio, frequência cardíaca (FC) e respiratória (FR). A AFE resultou em melhora da FR, FC, e saturação periférica de oxigênio em RNPT.

A repercussão da fisioterapia respiratória com a técnica de AFE sobre a hemodinâmica cerebral de recém-nascidos prematuros foi avaliada através do estudo de intervenção Bassani et al. (2016), no qual foram incluídos 40 RNPT com IG menor ou igual a 34 semanas. Contataram que a AFE não alterou o fluxo sanguíneo cerebral em recém-nascidos prematuros clinicamente estáveis, demonstrando ser segura e eficaz para esta faixa etária.

\section{Posição Prona}

O decúbito é uma estratégia capaz de melhorar a oxigenação arterial em pacientes com síndrome do desconforto respiratório (SDR) tratados com ventilação mecânica e PEEP (pressão expiratória final positiva) (OLIVEIRA et al., 2009). A posição prona elevada com coxins de gel nos ombros e nos quadris é recomendada para crianças com doença respiratória e índice de oxigenação alto, uma vez que melhoram a oxigenação. Os resultados desse decúbito iniciam nas primeiras 2 horas do posicionamento e se mantém durante as 12 horas subsequentes (JOHNSTON et al., 2012).

Atualmente, a ventilação mecânica com baixos volumes circulantes e a pressão positiva expiratória final é recomendada no tratamento da síndrome do desconforto respiratório. Entretanto, apesar disso, há pacientes que persistem hipoxêmicos e nos levam a considerar o uso de alternativas terapêuticas que visam melhorar a oxigenação arterial. $\mathrm{O}$ tratamento com posição prona é um deles. $\mathrm{O}$ uso da posição citada tem se mostrado uma técnica segura capaz de melhorar a oxigenação arterial dos recém-nascidos. Entretanto, até o momento, não se demonstrou efeito na sobrevida de pacientes com a síndrome (DOURADO et al., 2015).

A posição em decúbito ventral ou posição prona é aplicada em recém nascidos com síndrome de desconforto respiratório e tem demonstrado melhora da oxigenação em mais de $70 \%$ dos casos, além da melhora da heterogeneidade parenquimatosa, da complacência pulmonar, melhora da relação $\mathrm{V} / \mathrm{Q}$, diminuição do shunt intrapulmonar, melhora da vasoconstrição pulmonar, do recrutamento alveolar de áreas anteriormente dependentes da gravidade e redução da lesão pulmonar induzida pela ventilação mecânica (OLIVEIRA et al., 2008).

O estudo quase experimental de Oliveira et al. (2009) avaliou 12 RNPT com IG ao nascimento entre 28 e 36 semanas, comparando o efeito da posição PRONA e supina em RNPT pós SDR, respirando espontaneamente e em estado de sono ativo, sobre variáveis de padrão respiratório, movimento toracoabdominal e saturação periférica da hemoglobina pelo oxigênio. Evidenciaram que a posição PRONA 
promoveu redução significativa da assincronia toracoabdominal, sem influenciar o padrão respiratório e a saturação periférica de oxigênio.

A revisão de literatura de Dourado et al. (2015) identificou que a posição PRONA melhora vários parâmetros respiratórios, como frequência respiratória, saturação de oxigênio, volumes pulmonares, relação ventilação/ perfusão, contribuindo também para melhora do sincronismo toracoabdominal e diminuição do trabalho respiratório.

\section{Reequilíbrio Toracoabdominal (RTA)}

O método proporcionado pelo RTA pode ser empregado a pacientes prematuros que possuem disfunção respiratória. Frisa-se que a condição clínica não é limitante para o emprego de tal procedimento fisioterapêutico, haja vista que o manuseio alterará conforme as possibilidades de cada paciente (MIURA et al., 2012). A RTA consiste em uma terapia na qual promoverá a eliminação das secreções pulmonares e das vias aéreas superiores, pela ação dos músculos do trato respiratório promovendo a facilitação da tonicidade muscular do recém-nascido com síndrome do desconforto respiratório (ROSO et al., 2014).

A manobra deverá ser global, haja vista que os músculos não são relacionados apenas à respiração, mas à alimentação, captação sensorial, equilíbrio entre outras funções. O cerne do tratamento, desse modo, é reestabelecer essas funções, além do processo respiratório. Com isso, as principais funções de tal tratamento são: Redução do Esforço Muscular Ventilatório; Retirada de Secreções; Desbloqueio do Tórax; Reintegração das Atividades entre outras (VASCONCELOS et al., 2011).

No ensaio clínico randomizado com avaliador cego de Roussenq et al. (2013), avaliaram recémnascidos prematuros em UTIN com o objetivo verificar o efeito das manobras de RTA com relação às variáveis: frequência cardíaca, frequência respiratória, saturação periférica de oxigênio, desconforto respiratório e dor. Ao fim do estudo conclui que os RNs prematuros de baixo peso submetidos aos manuseios do método RTA apresentaram redução da frequência respiratória e do desconforto respiratório.

Achado controverso no estudo transversal de Steid et al. (2011), com 6 RNPT, idade gestacional de 32 semanas que não evidenciou melhora significativa das variáveis clínicas associada a realização da manobra. Constando apenas melhora do sincronismo toracoabdominal e do grau de desconforto respiratório após aplicação do método RTA. Fato explicado talvez pelo pequeno tamanho amostral.

\section{CONSIDERAÇÕES FINAIS}

A assistência fisioterapêutica a neonatos prematuros com SDR na UTIN é imprescindível para o sucesso da terapêutica e melhora progressiva da função pulmonar até a alta. $\mathrm{O}$ fisioterapeuta deve exercer um plano de tratamento integral e humanizado indo desde o ajuste ventilatório invasivo e não invasivo a execução de manobras terapêuticas, posicionamento e cuidados com a ocorrência de sequelas como hemorragia intraperiventricular e displasia broncopulmonar que podem comprometer o desenvolvimento neuropsicomotor e qualidade de vida após alta hospitalar. 


\section{REFERÊNCIAS}

ALDANA-AGUIRRE, J. C.; PINTO, M.; FEATHERSTONE, R. M.; KUMAR, M.. Less invasive surfactant administration versus intubation for surfactant delivery in preterm infants with respiratory distress syndrome: a systematic review and meta-analysis. ADC Fetal Neonatal, v.102, p.17-23, 2017. DOI: http://doi.org/10.1136/archdischild-2015-310299

ALMEIDA, T. S. O.; LINS, R. P.; CAMELO, A. L.; MELLO, D. C. C. L.. Investigação sobre os Fatores de Risco da Prematuridade: uma Revisão Sistemática. Revista Brasileira de Ciências da Saúde, Campina Grande, v.17, n.3, p.301-308, 2013.

ANGHEBEN, J. M. M.; MOURA, R. H.; ABREU, L. C. A.. Ventilação mecânica na síndrome do desconforto respiratório associado à reposição do surfactante. In: SARMENTO, G. J. V.. Princípios e práticas de ventilação mecânica em pediatria e neonatologia. Barueri: Manole, 2011. p.77-84.

AURILIA, C. C.; RICCI, I.; TANA, M.; TIRONE, C.; LIO, A.; GAMBACORTA, A.; PALADINI, A.; VENTO, G. G.. Management of pneumothorax in hemodynamically stable preterm infants using high frequency oscillatory ventilation: report of five cases. Italian Journal of Pediatrics, v.43, n.114, 2017. DOI: http://doi.org/10.1186/s13052-017-0436-y

BASSANI, M. A.; CALDAS, J. P. S.; NETTO, A. A.; MARBA, S. T. M.. Avaliação do fluxo sanguíneo cerebral de recém-nascidos prematuros durante a fisioterapia respiratória com a técnica do aumento do fluxo expiratório. Revista Paulista de Pediatria, Campinas, v.34, n.2, p.178-183, 2016.

BERNARDES, B. G.; MATTOS, F. G. M.. Proposta de protocolo de ventilação mecânica na síndrome do desconforto respiratório agudo. Revista UNILUS Ensino e Pesquisa, São Paulo, v.10, n.20, p.42-51, 2013.

BITTENCOURT, D.. Técnicas de fisioterapia respiratória na unidade de terapia intensiva neonatal. Revista Saúde Integrada, Santo Ângelo, v.10, n.19, p.1-15, 2017.

BURIA, G. C. O.. Análise da aplicabilidade e eficácia da manobra de bag Squeezing e seus efeitos fisiológicos. Monografia (Pós-Graduação) - Universidade Católica de Brasília, Brasília, 2013.

CABRAL, L. A.; VELOSO, M.. Comparação dos efeitos de protocolos de manuseio mínimo em parâmetros fisiológicos de prematuros submetidos à terapia do surfactante exógeno. Brazilian Journal of Physical Therapy, São Carlos, v.18, n.2, p.152-164, 2014. http://doi.org/10.1590/S1413$\underline{35552012005000154}$

CAVAGNOLI, A.; TAGLIETT, M.. Monitoramento da oxigenioterapia em recém-nascidos hospitalizados na unidade de terapia intensiva neonatal. FIEP BULLETIN, Paraná, v.84, 2014.

CEYLAN, A.; GEZER, S.; DEMIR, N.; TUNCER, O.; PEKER, E.; KIRIMI, E.. The importance of administration of early surfactant and nasal continuous positive airway pressure in newborns with respiratory distress syndrome. Turkish Archives of Pediatrics, v.49, n.3, p.192-197, 2014. DOI: http://doi.org/10.5152/tpa.2014.1624
CHAVES, C.. Repercussões da manobra de bag Squeezing com ou sem válvula reguladora de pressão positiva expiratória final em recém-nascidos pré-termo sob ventilação mecânica prolongada. Dissertação (Mestrado) Universidade de São Paulo, São Paulo, 2013.

DOURADO, F. P.; OLIVEIRA, N. R. G.; CAMPOS, I. R. M.. Posicionamento de prono na melhora da função respiratória de recém-nascidos pré-termo: uma revisão da literatura. Revista Movimenta, Goiânia, v.8, n.3, p.303-312, 2015

FIATT, M. P.; DAHER, B. R.; SANTOS, A. M.. Reversão de atelectasia em recém-nascido prematuro após uma sessão de fisioterapia respiratória: relato de caso. Revista HCPA, Porto Alegre, v.33, n.3, p.269-273, 2013.

FIORETTO, J. R.; REBELLO, C. M.. Ventilação oscilatória de alta frequência em pediatria e neonatologia. Revista Brasileira de Terapia Intensiva, Botucatu, v.21, n.1, p.96$103,2009$.

GEORGE, F. H. M.. Administração de surfactante pulmonar na síndrome de dificuldade respiratória do recém-nascido. Brasília: MS, 2012.

GONÇALVES, R. L.; TSUZUKI, L. M.; CARVALHO, M. G.. Aspiração endotraqueal em recém-nascidos intubados. Revista Brasileira de Terapia Intensiva, Manaus, v.27, n.3, p.284-292, 2015. DOI: http://doi.org/10.5935/0103507X.20150048

JOHNSTON, C.; ZANETTI, N. M.; COMARU, T.; RIBEIRO, S. N. S.; ANDRADE, L. B.; SANTOS, S. L. L.. Recomendação brasileira de fisioterapia respiratória em unidade de terapia intensiva pediátrica e neonatal. Revista Brasileira de Terapia Intensiva, São Paulo, v.24, n.2, p.119-129, 2012.

JÚNIOR, F. J. M. N.; SILVA, J. V. F.; FERREIRA, A. L. C.; RODRIGUES, P. A. R. A.. A síndrome do desconforto respiratório do recém-nascido: fisiopatologia e desafios assistenciais. Ciências Biológicas e da Saúde, Maceió, v.2, n.2, p.189-198, 2014.

LEÃO, E. V. V.; VIEIRA, M. E. B.; PEREIRA, A. S.. Perfil da utilização do CPAP na UTI neonatal e o protagonismo do fisioterapeuta. Revista Movimento, Goiânia, v.6, n.1, p.1984-4298, 2013.

LOBO, D. M. L.; CAVALCANTE, L. A.; MONT'ALVERNE, D. G. B.. Aplicabilidade das técnicas de bag Squeezing e manobra zeep em pacientes submetidos à ventilação mecânica. Revista Brasileira de Terapia Intensiva, Fortaleza, v.22, n.2, p.186-191, 2010.

MARTINS, R.; NUNES, P. M.; XAVIER, P. S.; WITTKOPF, P. G.; SCHIVINSKI, C. I. S.. Aspiração traqueal: a técnica e suas indicações. Arquivos Catarinenses de Medicina, Florianópolis, v.43, n.1, p.90-96, 2014.

NONA, J.; NOGUEIRA, M.; SILVA, T. C. P. V.; GARROTE, J. M.. Ventilação de Alta Frequência Oscilatória (VAFO). Lisboa: SPP, 2009.

OLIVEIRA, C. H. Y.; MORAN, C. A.. Ventilação mecânica não invasiva em recém-nascidos pré-termo com síndrome do 
desconforto respiratório. ConScientiae Saúde, São Paulo, v.8, n.3, p.485-489, 2009.

OLIVEIRA, L. R. C.; GARCIA, T. G.; PERES, V. G.; MAEDA, K. M.; OLIVEIRA, J. V.; ARAÚJO, J. P.; MOLINARI, C. V.; POLETTI, E. C.; JOSÉ, A.; CHIAVONE, P. A.. Ajustes da Pressão Positiva Expiratória Final Ideal na Síndrome do Desconforto Respiratório Agudo na Posição Prona. Revista Brasileira de Terapia Intensiva, Mairiporã, v.20, n.1, p.37-42, 2008.

OLIVEIRA, T. G.; REGO, M. A. S.; PEREIRA, N. C.; VAZ, L. O.; FRANÇA, D. C.; VIEIRA, D. S. R.; PARREIRA, V. F.. Prone position and reduced thoracoabdominal asynchrony in preterm newborns. Jornal de Pediatria, Belo Horizonte, v.85, n.5, p.443-448, 2009.

PICCOLI, A.; SOARES, C. R. S.; COSTA, G.; SILVEIRA, J. L.; FIATT, M. P.; CUNHA, R. S.. Perfil clínico de neonatos de muito baixos pesos internados em uma Unidade de Tratamento Intensivo Neonatal. Revista do Hospital de Clínicas e da Faculdade de Medicina, Porto Alegre, v.32, n.4, p.412-419, 2012.

REBELLO, C. M.; NACIF, L. F. A. N.; DEUTSCH, A. D. A.; PAES, A. T.. Momento do tratamento com surfactante em recémnascidos de muito baixo peso. Einstein, São Paulo, v.8, n.31, p.320-324, 2010.

ROSO, C. C.; COSTENARO, R. G. S.; RANGEL, R. F.; JACOBI, C. S.; MISTURA, C.; SILVA, C. T.; CORDEIRO, F. R.; PINHEIRO, A. L. U. P.. Vivências de mães sobre a hospitalização do filho prematuro. Revista de Enfermagem, Porto Alegre, v.4, n.1, p.47-54, 2014.

ROUSSENQ, K. R.; SCALCO, J. C.; ROSA, G. J.; HONÓRIO, G. J. S.; SCHIVINSKI, C. I. S.. Reequilíbrio toracoabdominal em recém-nascidos prematuros: efeitos em parâmetros cardiorrespiratórios, no comportamento, na dor e no desconforto respiratório. Revista Acta Fisiátrica, Florianópolis, v.20, n.3, p.118-123, 2013.

RUSCHEL, L.; NADER, P. J. H.. A doença da membrana hialina em prematuros de baixo peso. Revista da Associação Médica do Rio Grande do Sul, Porto Alegre, v.58, n.3, p.193197, 2014.

SÁ, F. E.; FROTA, L. M. C. P.; OLIVEIRA, I. F.; BRAVO, L. G.. Estudo sobre os efeitos fisiológicos da Técnica de Aumento do Fluxo Expiratório Lento em prematuros. Revista Fisioterapia \& Saúde Funcional, Fortaleza, v.1, n.1, p.16-21, 2012.

SILVA, D. M.; CHAVES, E. M. C.; FARIAS, L. M.; LÉLIS, A. L. P. A.. Uso de pressão positiva contínua das vias aéreas em recém-nascidos: conhecimento da equipe de enfermagem. Revista Rene, Fortaleza, v.11, p.195-203, 2010.
SILVA, J. D.; MARON-GUTIERREZ, T.; ROCCO, P. R. M.; GARCIA, C. S. N. B.. Terapia com surfactante na síndrome do desconforto respiratório agudo: prós e contras. Pulmão RJ, Rio de Janeiro, v.18, n.3, p.148-154, 2009.

SOUZA, K. C. L.; CAMPOS, N. G.; SANTOS, F. F. U. J.. Perfil dos recém-nascidos submetidos à estimulação precoce em uma unidade de terapia intensiva neonatal. Revista Brasileira de Promoção à Saúde, Fortaleza, v.26, n.4, p.523-529, 2013.

STEIDL, E. M. S.; KOOP, L.; ANTUNES, V. P.. Avaliação das variáveis clínicas em recém-nascidos pré-termo submetidos à ventilação mecânica pré e pós hiperinsuflação manual. Revista Contexto e Saúde, ljuí, v.9, n.16, 2009. DOI: https://doi.org/10.21527/2176-7114.2009.16.147-150

STEIDL, S. E. M.; TASSINARI, C. C. R.; ANTUNES, V. P.. Influência do método reequilíbrio toracoabdominal em recém-nascidos pré-termos pós-síndrome do desconforto respiratório. Revista Contexto \& Saúde, Porto Alegre, v.11, n.21, 2011.

STEVENS, T. P.; HARRINGTON, E. W.; BLENNOW, M.; SOLL, R. F.. Early surfactant administration with brief ventilation vs. selective surfactant and continued mechanical ventilation for preterm infants with or at risk for respiratory distress syndrome. Cochrane Database System Review, Chichester, v.17, n.4, 2007. DOI: http://doi.org/10.1002/14651858.CD003063.pub3

UTYAMA, J. A.; ROMPINELLI, V. O.; FREITAS, N. M. M.; DANTAS, E. O.; RULLO, V. E. V.. Intercorrências respiratórias em recém-nascidos prematuros de baixo peso. Revista UNILUS Ensino e Pesquisa, Santos, v.13, n.30, p.2318-2083, 2016.

VASCONCELOS, G. A. R.; ALMEIDA, R. C. A.; BEZERRA, A. L.. Repercussões da fisioterapia na unidade de terapia intensiva neonatal. Revista Fisioterapia em Movimento, Recife, v.24, n.1, p.65-73, 2011.

VIANA, C. C.; NICOLAU, C. M.; JULIANA, R. C.; CARVALHO, W. B.; KREBS, V. L.. Efeitos da hiperinsuflação manual em recém-nascidos pré-termo sob ventilação mecânica. Revista Brasileira de Terapia Intensiva, São Paulo, v.28, n.3, p.341347, 2016. DOI: http://doi.org/10.5935/0103507X.20160058

YAGUI, A. C. Z.; VALE, L. A. P. A.; HADDAD, L. B.; PRADO, C.; ROSSI, F. S.; DEUTSCH, A. D. A.; REBELLO, C. M.. Bubble CPAP versus CPAP with variable flow in newborns with respiratory distress: a randomized controlled trial. The Journal of Pediatrics, Rio de Janeiro, v.87, n.6, p.499-504, 2011. DOI: http://doi.org/10.2223/JPED.2145

A CBPC - Companhia Brasileira de Produção Científica (CNPJ: 11.221.422/0001-03) detém os direitos materiais desta publicação. Os direitos referem-se à publicação do trabalho em qualquer parte do mundo, incluindo os direitos às renovações, expansões e disseminações da contribuição, bem como outros direitos subsidiários. Todos os trabalhos publicados eletronicamente poderão posteriormente ser publicados em coletâneas impressas sob coordenação da Sustenere Publishing, da Companhia Brasileira de Produção Científica e seus parceiros autorizados. Os (as) autores (as) posteriormente ser publicados em coletâneas impressas sob coordenação da Sustenere Publishing, da Companhia Brasileira de Produção Cientifica e seus parce
preservam os direitos autorais, mas não têm permissão para a publicação da contribuição em outro meio, impresso ou digital, em português ou em tradução. 